\title{
Developmental Origins of Obesity - Genetic and Epigenetic Determinants
}

\author{
Benjamin Rokholm,\#, Camilla Schou Andersen ${ }^{\#}$ and Thorkild I.A. Sørensen \\ Institute of Preventive Medicine, Centre for Health and Society, Copenhagen University Hospital, DK-1357 \\ Copenhagen, Denmark
}

\begin{abstract}
Background: Childhood obesity is a major public health concern. The importance of genes in development of obesity is evident from twin and family studies. Moreover, epigenetic events in early life may play an important role in obesity etiology. Aim: We aimed to outline and discuss the role of genetic and epigenetic influences on development of obesity in early life from around birth and into adolescence. Methods: We included a series of interesting contributions on the area. First, the topic was addressed on the relative influence of genes and environment. Next, we turned to the significance of epigenetic events. Results: Genetic influences play an important role in individual differences in body fatness throughout childhood and adolescence, with an increasing heritability with age. In addition, environmental factors can influence the epigenetic regulation, making the individual more susceptible to develop obesity. Conclusion: Our knowledge of genetic and epigenetic contributions to obesity development has increased dramatically during the last century. Future studies and technological advnces, will undoubtedly reveal even more complicated pathways that have to be considered when trying to prevent the dramatic worldwide increase in obesity.
\end{abstract}

Keywords: Genetic factors, epigenetic regulation, obesity, childhood.

\section{INTRODUCTION}

The prevalence of obesity has increased at epidemic rates in most parts of the world in the last decades and in United States the most recent data show, that more than a third of all men and women are obese [1,2]. At the same time it has been estimated that 30-45 million children are obese worldwide (www.world-heart-federation.org). Childhood obesity is a major public health concern. The condition is associated with psychosocial complications such as social isolation, low self-esteem and body image as well as medical conditions including type 2 diabetes, hypertension, non-alcoholic fatty liver disease, gastroesophageal reflux and gallstones [3, 4]. In addition, studies show that childhood obesity also leads to weight problems later in life [5].

The understanding of how genes influence the development of obesity has greatly improved in the recent years. This is mainly due to important technological advances allowing genome-wide search for genes associated with obesity phenotypes - the perhaps most prominent result being the discovery of the FTO gene, which is associated with body mass index (BMI) and risk of obesity.

The aim of this article is to outline the role of genetic and epigenetic events in development of obesity in early life. This topic can naturally not be covered comprehensively in one article. Instead a series of interesting findings and theories on the area will be highlighted. First we will approach the subject from the perspective of twin and family studies in which the relative influence of genes and

\footnotetext{
*Address correspondence to this author at the Institute of Preventive Medicine, Øster Søgade 18,1, DK-1357 Copenhagen K, Denmark; Tel: + 45 3338 3817; Fax: +45 3332 42 40; E-mail: br@ipm.regionh.dk

${ }^{\#}$ The two authors equally contributed to the manuscript.
}

environmental factors in a population can be investigated. Next we will turn to the possible role of epigenetic events in early life in development of obesity. Epidemiological evidence increasingly suggests that exposure to certain environmental factors early in development can affect the phenotype later in life. In addition, some of these environmental effects seem to be passed on through subsequent generations. Increasing numbers of excellent reviews have already been published, describing the detailed mechanisms of epigenetic regulation and the suggested associations to disease susceptibility [6-14]. Therefore, in this review we will give a short overview of the current knowledge from animal and human studies on the possible link between genetic inheritance, epigenetic regulation and childhood obesity.

\section{LEARNINGS FROM TWIN STUDIES}

In the following we will discuss contributions from twin studies to the knowledge of developmental origins of obesity. The stages of development will be covered broadly from birth into childhood and during pubertal transition. However, the intention is not to review all relevant literature, but rather to outline some of the main findings on the area. A wide variety of quantitative genetic studies exist including different twin, family and adoption designs ${ }^{1}$. Nevertheless, we will primarily discuss findings from twin studies, from which most of the contributions origin. The main focus will be on genetic influences, but evidence on environmental determinants from twin studies, will be considered when appropriate.

\footnotetext{
${ }^{1}$ In principle all relations in which either environmental or genetic factors (or both) are correlated, can contribute with information on influence from these sources, if combined correctly [15].
} 


\section{The Classical Twin Design}

By studying resemblance in a trait within pairs of different types of family members, or individuals sharing the same environment, it is possible to segregate the influence of genes from the influence of the environment. In the case of twins, we can estimate the environmental and genetic contribution to phenotypic variance by calculating trait correlations for monozygotic (MZ) and dizygotic (DZ) twin pairs, respectively. This is because MZ twins share all their genetic material, while DZ twins, on average, share $50 \%$ of their segregating genes. Thus, assuming that $\mathrm{MZ}$ and $\mathrm{DZ}$ twin pairs share an equally similar environment, a higher correlation between MZ than DZ twins gives evidence a genetic contribution to the trait [16]. Through twin and family studies we can identify four sources of phenotypic variation.

The variation can be caused by,

1. $\mathbf{A}=$ additive genetic effects, which is the sum of the effects of all genetic loci influencing the trait (the additive genetic component),

2. $\quad \mathbf{D}=$ dominant genetic effect, (the dominant genetic component) which refers to the effects of gene-gene interaction at a single locus (dominance) and between genes at different loci (epistasis) ${ }^{2}$,

3. $\mathbf{C}=$ environmental factors shared by both members in a pair (the common/shared environmental component),

4. $\quad \mathbf{E}=$ environmental factors unique to each member in a pair (the unique/unshared environmental component)

In the classical twin design, in which twins are reared together, the $\mathrm{C}$ and $\mathrm{D}$ components cannot be estimated simultaneously. Thus, either the ACE or the ADE model is chosen, depending on the goodness of fit. Heritability is often used as a measure of genetic influence on a trait. It refers to the proportion of total variation in the trait, attributable to genetic differences between individuals in a population. A heritability of e.g. 0.4 means that $40 \%$ of the total phenotypic variation is caused by genetic factors and 60 $\%$ by environmental influences.

\section{Maximising the Utility of Twin Studies}

The primary target of twin and family studies has mostly been to estimate heritability of a trait in a given population. Nevertheless, the quantitative genetic approach offers a wide range of possibilities. Firstly, not only the genetic influences should be considered. In order to fully take advantage of the method, we should also assess the remaining components, i.e. common environmental and unique environmental variation. Secondly, in addition to considering a single snapshot of the distribution of phenotypic variance, we can follow a population of twins from early childhood into adolescence or compare cross-sectional studies of children at different ages. This enables us to investigate possible changes in genetic and environmental influences during a life course and to clarify to what extend an association between BMI in childhood and adulthood is caused by genes and the environment,

\footnotetext{
${ }^{2}$ In the case of closely linked interacting loci, which are segregated together, the effects are modelled as additive genetic effects since the genes interacting, in this case, form a unit.
}

respectively [16]. In the following we will take a look at some of the evidence available from twin studies at present time.

\section{Genetic and Environmental Factors in Childhood Obesity}

In a recent review of twin and adoption studies, genetic factors had a strong effect on BMI variation at all ages in childhood [17]. In the context of the current paper it is relevant to also consider determinants for birth weight, which has been found to be associated with adult BMI [18]. In two independent twin studies Dubois et al. and Vlietinck et al. estimated the heritability of weight at birth to be 0.4 $[19,20]$. Demerath et al. studied infant weight in nuclear and extended families and found that a higher proportion of the variance explainable by additive genetic effects (heritability $=0.61-0.95$ ) [21]. Haworth et al. studied the later stages of development and found evidence that heritability of BMI increased over childhood from 0.56 at 4 years to 0.78 at 11 years [22]. Likewise, a longitudinal study of Dutch twins showed that heritability increases from age 3 to age 10 in boys and age 12 in girls [23]. The study also found a very similar age trend in the effect of the FTO gene on BMI. The age trends suggested by these studies are illustrated in Fig. (1). However, in a Swedish longitudinal study, Silventoinen et al. found consistently high estimates of heritability (between 0.83 and 0.92) in male twins from birth to age 18, with no sign of a systematic age pattern [24].

The source of heterogeneity between twin studies in estimates of heritability is yet largely unknown. Multiple factors varying across populations are likely to influence heritability of body weight and BMI, in addition to age. Furthermore, it is important to recall that since heritability is a proportion, it is also influenced by environmental factors. Hence, an age trend in heritability must be interpreted carefully, since it could reflect changes in environmental influences as well. In order to more comprehensively elucidate the issue of a possible age pattern, a meta-regression analysis of studies reporting heritability estimates in childhood could be conducted.

As mentioned, it can be informative to also consider the environmental components in twin and family studies. Most studies report the ACE or AE model to have the best fit on data. This indicates that unshared environmental factors, denoted by E, significantly contribute to BMI variation at all ages. On the other hand, the $\mathrm{C}$ component shows a more inconsistent pattern. Silventoinen et al. systematically reviewed available evidence from twin and adoption studies, which indicated that common environmental factors substantially affected BMI in early childhood, but that this influence disappeared in adolescence [17]. This finding concurs with a prospective study of Finnish twins in which environmental effects shared by siblings were 0.15-0.24 among 11-12 and 14-year-old boys and girls, but no longer discernible at 17 years of age [25].

A more sophisticated usage of the twin and family approach enables us to estimate the overlap in genetic and environmental factors at different periods in childhood. In this way we can investigate whether genetic and environmental factors influencing body weight at one time are also 


\section{Age trend in heritability of BMI}

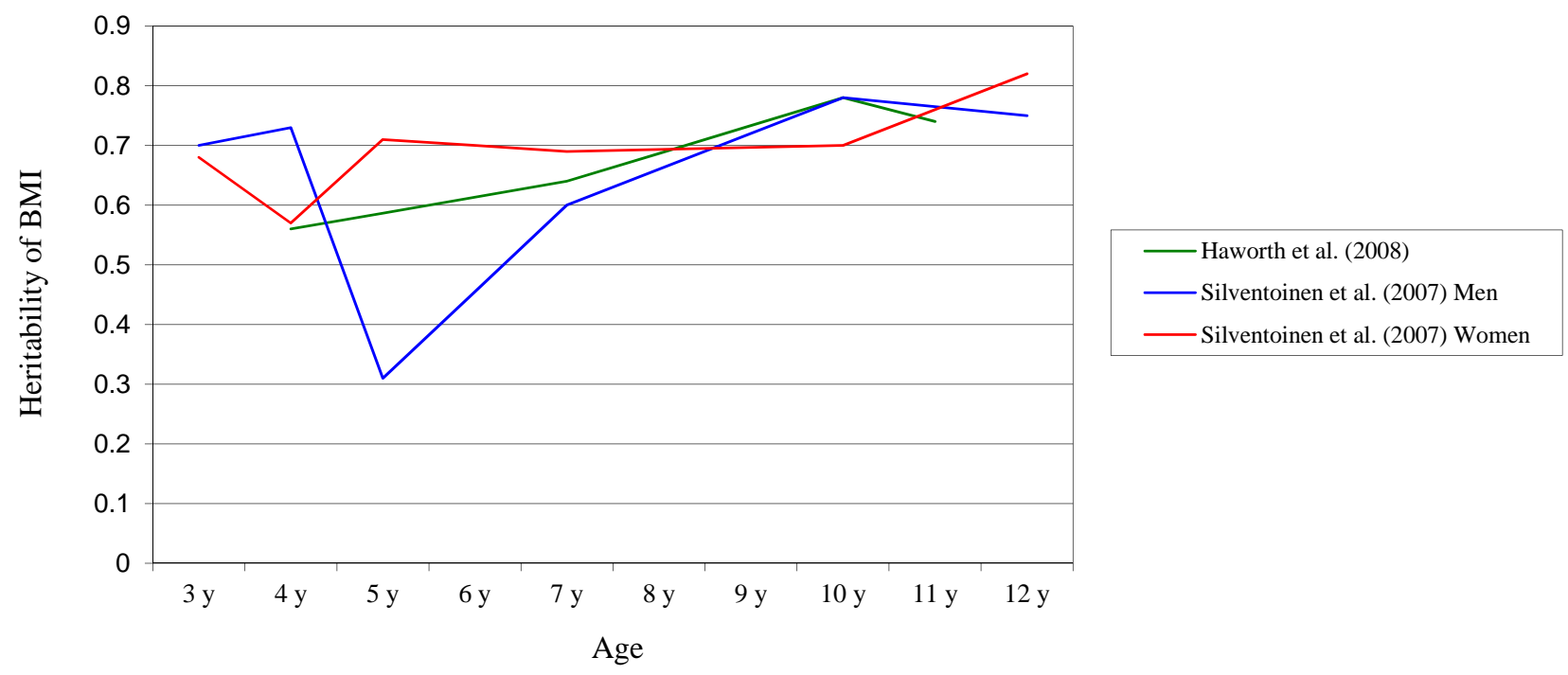

Fig. (1). Age trend in heritability of BMI in childhood.

active at later developmental stages. This is relevant since previous studies suggest that adiposity in childhood is a strong predictor of adulthood obesity [26, 27]. Thus, if a large proportion of the genetic influences on adiposity in childhood remain important in adulthood, we can hypothesise that genetic factors are important for the association between childhood and adulthood obesity. The method was applied to Swedish male twins in a longitudinal study from birth to 18 years of age [24]. Correlations between BMI at age 18 and ponderal index at birth and BMI from age 1 up to age 17 were calculated. The correlations increased with increasing age from 0.32 between 1 and 18 years to 0.91 between 17 and 18 years. Almost all of this tracking could be explained by additive genetic factors (81-95\%) and the remaining part by unique environmental correlation. In other words, the carry-over effect during growth was almost entirely caused by genetic factors. Nevertheless, it is interesting to note that a low but significant unique environmental correlation was found between ponderal index at birth and BMI at age 18. This indicates that the intra-uterine environment may be important for adult BMI, which is also supported by previous findings [28].

From a public health perspective, knowledge on the specific factors constituting the genetic and environmental components is crucial. The fact that genetic factors are responsible for the majority of BMI variation does not necessarily imply, that the resulting phenotype is beyond our influence. It is important to note that behavioural patterns such as eating behaviour and physical activity, which are often thought to be related to BMI, have a strong genetic component [29, 30]. Insofar as diet and physical activity is affecting BMI, part of the heritability of BMI is mediated through these factors. Hence, since eating habits and exercise can also be altered deliberately, genetic influences are not necessarily predetermined.

\section{Drawing Conclusions from Twin and Family Studies}

An important strength of twin and family studies is in areas in which knowledge of specific causal mechanisms is still at an early stage. The findings can be used as a point of orientation for future studies by contributing with information on what type of factors are important. As an example, Wardle et al notes that since twin studies most often report insignificant or low estimates of the common environmental component, it is likely that the family environment is of low significance in the development of obesity [31]. Based on these results, the authors recommend caution in making parents responsible for obesity in their children. Likewise, measures of heritability can be utilized at a molecular genetic level. If heritability is high in a given population at a given time the potential of locating candidate genes is probably higher.

However, we must be careful in our interpretation of results from twin and family studies. Firstly, the size of the genetic or environmental components does not always reflect the importance of these factors. This is because the size of the components depends on differences in exposure between individuals. If all individuals in a population are equally exposed this will not cause variation even though the exposure may be the main cause of an adverse health condition. In this case the exposure, although important, will not be measurable. Secondly, when the size of the components are reported as proportions, which is often the case, the genetic contribution to total variance will depend on the environmental contribution and vice versa.

\section{THE ROLE OF EPIGENETIC EVENTS}

To a large extent, our genes determine our body weight, as just described in the previous sections. However, by nature, the dramatic increase in obesity prevalence cannot 
solely be explained by our genes, due to the comparatively short period of 40-50 years [32]. Therefore, complex interactions between the genome and perturbations in our environment have to be taken into account when considering susceptibility to gain excess of weight. Especially insults during sensitive periods of development in the perinatal life, can induce detrimental and long-lasting effects [33], and has been termed the fetal origins hypothesis [34]. These insults are thought, in part, to involve epigenetic regulation, defined as mitotically and/or meiotically heritable changes in gene function, without any changes in DNA sequence [11].

\section{Short Definition of Epigenetic Regulation}

Before we consider how epigenetic modulation possibly can be involved in the aetiology of obesity development, it is important to bear in mind that epigenetic regulation is an essential process in normal development that occurs all through life. It is required to achieve stable expression or repression of genes in specific cell types or at specific time points and constitutes the link between genotype and phenotype [35]. Epigenetics encompasses two major modifications of DNA or chromatin: DNA methylation and modification of the histones tails including methylation, acetylation and phosphorylation [14]. The best-characterized form of epigenetic marking, is methylation of the cytosine residues of DNA, especially in $\mathrm{CpG}$ dinucleotides present in regions called $\mathrm{CpG}$ islands. DNA methylation is generally associated with reduced transcriptionally activity, not only through decreased binding of transcription factors but also by attracting methyl-CpG-binding proteins that act as transcriptional repressors. The different modifications of histones, in majority methylation or acetylation, cause condensation or relaxation of the local chromatin structure, thereby regulate the access to the underlying DNA [36].

Some of the different types of genes and processes under the influence of epigenetic control include X-chromosome inactivation and imprinted genes [37]. X-chromosome inactivation is the key process happening in somatic cells in female embryos, where one of the $\mathrm{x}$-chromosomes is randomly inactivated by methylation [38]. The imprinting of app. $1 \%$ of our autosomal genes, meaning that only the maternally or the paternally derived allele are involved in the expressed gene product, plays significant roles in development and cellular proliferation $[39,40]$.

\section{Disturbances in Epigenetic Mechanisms}

The cell cycle maintenance of differential DNA methylation and its associated chromatin features requires a complex interplay between many different enzymes. This constant gene repression or activation is governed to a large extent, by intrinsic factors, such as methyltransferases, and these mechanisms most often function faithfully. However, different environmental perturbations sometimes destroy this perfectly maintenance of epigenetic patterns, leading to increased susceptibility for later disease [14]. In rodents, grooming behaviour from the mother during the pups first weeks of life, can affect the methylation status of the glucocorticoid receptor gene. Thereby, absence of appropriate maternal care influences the offspring's stress response later in life [41]. This could possibly also influence utilization of diet, due to the important role of glucocorticoid in macro- nutrient metabolism. Other influences from the environment, that have received a huge amount of interest is nutritional status, since it seems to affect development of adipose tissue, appetite regulation and metabolic processes [36].

\section{Epigenetic Modulation Involved in Weight Regulation}

Most studies conveying results about nutritional interruptions in the epigenetic control have been performed in animals. Diet-induced obesity has in several animal studies been associated to epigenetic changes in genes associated to body weight regulation. In adult rats, intakes of high proportions of fat induced increased weight in the animals and methylation in the leptin gene [42]. These effects can be counteracted by supplementation of ascorbic acid with altered expression of genes in rodent adipose tissue [43]. In young rats, overfeeding either in utero [44] or during neonatal life [45] induced changed methylation patterns in the appetite regulating hormones in the hypothalamus. Some of the effects persisted after puberty with increased weight, and increased insulin- and leptin- and triglyceride levels [44].

In sheep, increased nutrition in prenatal life induced permanent changes in the appetite regulatory system and the adipocytes resulting in increased adiposity 30 days after birth [46]. In non-human primates, maternal high-fat diet during pregnancy induced a variety of modifications in chromatin structure in the offspring, followed by increased expression of several hepatic and metabolic enzymes. These changes were associated with elevated levels of triglycerides, histological correlates of non-alcoholic fatty liver disease and increased body weight in the fetus [47] and in the juveniles [48]. In contrary to excess energy availability, different kind of maternal restricted diets have also been shown to possible lead to a disturbed metabolism in the offspring. A study in rats by Lillycrop and colleagues showed that maternal exposure to a protein-restricted diet during pregnancy induced hypomethylation in the juveniles' hepatic PPAR $\alpha$ promoter, with persistent effect into adulthood. However, the adverse effects of a low-protein diet could be prevented by also feeding the pregnant rats the methyl donor folic acid [49]. In other studies, rats exposed to maternal undernutrition during the fetal period, had elevated expression of the leptin gene and increased adiposity in adult life. If these undernourished rats however were treated with leptin during the neonatal period, it reversed the adverse effects from in utero undernutrition [50]. These findings were explained by leptins important role in the development of nuclear projections in hypothalamus, involved in regulating body weight [50].

Another dietary compound, the phytoestrogen genistein, found in soy and soyproducts has been involved in alterations in the mouse epigenome. In a study by Dolinoy and colleagues, in utero exposure to genistein, resulted in hypermethylation of the agouti gene. This gave a different coat color and decreased incidence of adult onset of obesity [51].

It is important to recognize that many of the developmental events involved in metabolism and energy homeostasis occur postnatally in the rodent, whereas these often occur in utero in humans. Therefore, it can be difficult to extrapolate results from animal studies into human settings. 
Recent evidence from human studies, do however support that epigenetic modulation can be involved in weight regulation [52]. Campión and colleagues, have in a current review, reported a list of more than 20 human genes possibly regulated by epigenetic mechanisms involved in development of obesity. These genes are implicated in different physiological processes, such as adipogenesis, inflammation, energy and fat metabolism, and insulin signalling and resistance [53].

Besides nutritional factors, evidence about detrimental effects from endocrine disruptors has been suggested. In utero or neonatal exposure to bisphenol A (BPA), a manmade chemical used in the manufacture of plastic, is associated among other things, with decreasing DNA methylation of the Agouti gene and higher body weight in mice. However, maternal dietary supplementation, with either methyl donors like folic acid or the phytoestrogen genistein, did negate the negative effects of BPA on the epigenome [54].

Thus, compelling evidence is presented that different environmental factors can possibly affect individuals phenotype throughout the life span, by stably altering the epigenome. During gestation however, the epigenome is most vulnerable to environmentally induced dysregulation, because the DNA synthetic rate is high and the DNA methylation patterning required for normal tissue development is established during this period [8]. Interestingly, the detrimental maternal effects can sometimes be counteracted by e.g. maternal dietary supplements. These findings strongly support increased investigation of nutritional supplementation as a parental preventive approach to counteracting environmental influences on the epigenome [9].

Much of the evidence about epigenetic regulation possibly involved in development of obesity comes from specific effects on certain genes already known to be involved in metabolic mechanism. Additional new perspectives to how epigenetic effects may influence obesity development in childhood, comes from the Prader-Willi Syndrome [32]. This syndrome is associated with cognitive impairments, uncontrollable appetite and severe obesity within the first 6 years of life. These dysfunctions results from altered genomic imprinting and thereby disturbed expression of gene products. Therefore, it is plausible that subtle imprinting of yet unidentified genes may also contribute to individual variations in body mass $[32,55]$.

\section{Transgenerational Effects}

The possibility that environmental factors can exert effects through generations is receiving intense interest. However, the idea of epigenetic regulation with transgenerational effects could be involved in the metabolic syndrome is not new. Some Swedish studies conducted for more than 100 years ago, suggested that men who experienced access to plenty of foods and nutrients during pre-pubertal stage, would have grandsons with higher risk of dying from diabetes [56]. This is in agreement with much later studies, where mortality of diabetes and cardiovascular disease were influenced by the nutritional state of parents and grand parents [57]. Data from the Dutch Famine study further supports the possible effects through generations. Mothers who experienced famine in $3^{\text {rd }}$ trimester had children with lower birth weight and increased risk of insulin resistance. In addition, grandchildren of mothers experiencing famine in early pregnancy also had reduced birth weight, independent of any effect on the first generation [58, 59]. Since these transgenerational effects cannot easily be explained by genetic mutations, epigenetic regulation has been suggested to be involved [36].

If influences from the environment are carried on to children, grandchildren and great-grandchildren, the effects are very serious. Obviously, transgenerational effects must work through interacting and complicated pathways, and there are still a lot of details about possible mechanisms that needs to be unraveled. The possibility that parental lifestyle factors influence the susceptibility of obesity for several generations will have dire consequences and more research is imperative in order to find ways to prevent these potentially vicious cycles.

\section{DISCUSSION}

Twin and family studies show evidence of a considerable genetic component to individual differences in weight at birth and BMI at all periods in childhood. Some studies suggest an age trend in heritability and the importance of shared environmental factors. Two independent studies suggest that the relative importance of genetic factors increases from early to late childhood. A parallel tendency was found on a molecular genetic level in the FTO gene, which is associated with BMI and the risk of obesity. The effect of FTO on BMI was increasing over childhood in a pattern similar to the increase in heritability of BMI. Twin studies are also informative regarding environmental influences on adiposity. At least two studies suggest that common environmental factors are important in early childhood, and subsequently vanish in adolescence. Unshared environmental effects, on the other hand, remains important at all ages. Tracking of BMI in longitudinal twin studies can give insight into overlapping genetic and environmental factors at different periods of development. A Swedish male twin study reported that almost all the tracking $(81-95 \%)$ could be explained by additive genetic factors and the remaining part was caused by unshared environmental factors. This suggests that associations between childhood and adulthood BMI primarily is genetically determined.

Although the focus in genetic epidemiological research in the recent years has moved to molecular genetics studies, quantitative genetic methods undoubtedly still have more to offer. Twin and family studies show that the majority of variation in BMI is caused by genetic factors. Nevertheless, studies on candidate genes have only accounted for a few percentages of the total genetic variation. This calls for new ways of understanding the role of genes in obesity development. In this exploratory phase twin and family studies are ideal, since hypotheses can be tested without restricting the analyses to particular genetic loci. More research using the twin and family approach should therefore be encouraged.

Obesity is certainly a multifaceted disease with complex interactions between genetic traits, epigenetic regulation, environmental factors, hereof the amount of influence and 
the timing of exposure and on top of that, inter-individual susceptibility to evolve disease. Even though, extensive animal models and human epidemiological data support the hypothesis about early environmental factors influencing on later susceptibility for obesity, our understanding of how genes and epigenetics are involved in the etiology of obesity has just begun. In the next couples of years, more sophisticated technology will undoubtedly unravel more knowledge about the underlying biological mechanisms. With more knowledge, it has been hypothesized, that personalized medicine or novel diets could be used to prevent or reverse the detrimental epigenetic effects leading to overweight and obesity. However, more information about the complex interplay between nutritional diversity, individual genetic backgrounds and intestinal physiological environments where food is metabolized is detrimental before medicine or nutrition can be used to abolish any adverse effects $[32,60]$.

\section{REFERENCES}

[1] Ford ES, Mokdad AH. Epidemiology of obesity in the Western Hemisphere. J Clin Endocrinol Metab 2008; 93(11 Suppl 1): S1S8.

[2] Ogden CL, Yanovski SZ, Carroll MD, Flegal KM. The epidemiology of obesity. Gastroenterology 2007; 132(6): 2087102.

[3] Freedman DS, Dietz WH, Srinivasan SR, Berenson GS. The relation of overweight to cardiovascular risk factors among children and adolescents: the Bogalusa Heart Study. Pediatrics 1999; 103(6 Pt 1): 1175-82.

[4] Guzzaloni G, Grugni G, Minocci A, Moro D, Morabito F. Liver steatosis in juvenile obesity: correlations with lipid profile, hepatic biochemical parameters and glycemic and insulinemic responses to an oral glucose tolerance test. Int J Obes Relat Metab Disord 2000; 24(6): 772-6.

[5] Eriksson J, Forsen T, Osmond C, Barker D. Obesity from cradle to grave. Int J Obes Relat Metab Disord 2003; 27(6): 722-7.

[6] Bird A. Perceptions of epigenetics. Nature 2007; 447(7143): 396-8.

[7] Callinan PA, Feinberg AP. The emerging science of epigenomics. Hum Mol Genet 2006; 15 Spec No 1: R95-101.

[8] Jaenisch R, Bird A. Epigenetic regulation of gene expression: how the genome integrates intrinsic and environmental signals. Nat Genet 2003; 33 (Suppl): 245-54.

[9] Jirtle RL, Skinner MK. Environmental epigenomics and disease susceptibility. Nat Rev Genet 2007; 8(4): 253-62.

[10] Klose RJ, Bird AP. Genomic DNA methylation: the mark and its mediators. Trends Biochem Sci 2006; 31(2): 89-97.

[11] Nafee TM, Farrell WE, Carroll WD, Fryer AA, Ismail KM. Epigenetic control of fetal gene expression. BJOG 2008; 115(2): 158-68.

[12] Wadhwa PD, Buss C, Entringer S, Swanson JM. Developmental origins of health and disease: brief history of the approach and current focus on epigenetic mechanisms. Semin Reprod Med 2009; 27(5): 358-68.

[13] Waterland RA, Jirtle RL. Early nutrition, epigenetic changes at transposons and imprinted genes, and enhanced susceptibility to adult chronic diseases. Nutrition 2004; 20(1): 63-8.

[14] Waterland RA, Michels KB. Epigenetic epidemiology of the developmental origins hypothesis. Ann Rev Nutr 2007; 27: 363-88.

[15] Neale MC, Maes HHM. Methodology for Genetic Studies of Twins and Families. Kluwer Academic Publishers B.V. Dordrecht, The Netherlands; 2006.

[16] Neale MC, Maes HHM. Methodology for genetic studies of Twins and Families. Kluwer Academic Publishers B.V. Dordrecht, The Netherlands; 2006.

[17] Silventoinen K, Rokholm B, Kaprio J, Sørensen TIA. Genetic and environmental influences on childhood obesity: systematic review of twin and adoption studies. Int J Obes 2009; (Accepted, awaiting press).

[18] Martorell R, Stein AD, Schroeder DG. Early nutrition and later adiposity. J Nutr 2001; 131(3): 874S-80S.
[19] Dubois L, Girard M, Girard A, Tremblay R, Boivin M, Perusse D. Genetic and environmental influences on body size in early childhood: a twin birth-cohort study. Twin Res Hum Genet 2007; 10(3): 479-85.

[20] Vlietinck R, Derom R, Neale MC, et al. Genetic and environmental variation in the birth weight of twins. Behav Genet 1989; 19(1): 151-61.

[21] Demerath EW, Choh AC, Czerwinski SA, et al. Genetic and environmental influences on infant weight and weight change: the Fels Longitudinal Study. Am J Hum Biol 2007; 19(5): 692-702.

[22] Haworth CM, Carnell S, Meaburn EL, Davis OS, Plomin R, Wardle J. Increasing Heritability of BMI and Stronger Associations With the FTO Gene Over Childhood. Obesity (Silver Spring) 2008; 16(12): 2663-8.

[23] Silventoinen K, Bartels M, Posthuma D, et al. Genetic regulation of growth in height and weight from 3 to 12 years of age: a longitudinal study of Dutch twin children. Twin Res Hum Genet 2007; 10(2): 354-63.

[24] Silventoinen K, Pietilainen KH, Tynelius P, Sorensen TI, Kaprio J, Rasmussen F. Genetic and environmental factors in relative weight from birth to age 18: the Swedish young male twins study. Int J Obes (Lond) 2007; 31(4): 615-21.

[25] Lajunen HR, Kaprio J, Keski-Rahkonen A, et al. Genetic and environmental effects on body mass index during adolescence: a prospective study among Finnish twins. Int J Obes (Lond) 2009; 33(5): 559-67.

[26] Parsons TJ, Power C, Logan S, Summerbell CD. Childhood predictors of adult obesity: a systematic review. Int J Obes Relat Metab Disord 1999; 23 (Suppl 8): S1-107.

[27] Sun SS, Liang R, Huang TT, et al. Childhood obesity predicts adult metabolic syndrome: the Fels Longitudinal Study. J Pediatr 2008; 152(2): 191-200.

[28] Rogers I. The influence of birthweight and intrauterine environment on adiposity and fat distribution in later life. Int J Obes Relat Metab Disord 2003; 27(7): 755-77.

[29] Joosen AM, Gielen M, Vlietinck R, Westerterp KR. Genetic analysis of physical activity in twins. Am J Clin Nutr 2005; 82(6): 1253-9.

[30] Teucher B, Skinner J, Skidmore PM, et al. Dietary patterns and heritability of food choice in a UK female twin cohort. Twin Res Hum Genet 2007; 10(5): 734-48.

[31] Wardle J, Carnell S, Haworth CM, Plomin R. Evidence for a strong genetic influence on childhood adiposity despite the force of the obesogenic environment. Am J Clin Nutr 2008; 87(2): 398-404.

[32] Stoger R. Epigenetics and obesity. Pharmacogenomics 2008; 9(12): 1851-60.

[33] Junien C, Nathanielsz P. Report on the IASO Stock Conference 2006: early and lifelong environmental epigenomic programming of metabolic syndrome, obesity and type II diabetes. Obes Rev 2007; 8(6): 487-502.

[34] Barker DJ. The origins of the developmental origins theory. J Intern Med 2007; 261(5): 412-7.

[35] Li E. Chromatin modification and epigenetic reprogramming in mammalian development. Nat Rev Genet 2002; 3(9): 662-73.

[36] Gluckman PD, Hanson MA, Beedle AS. Non-genomic transgenerational inheritance of disease risk. Bioessays 2007; 29(2): 14554.

[37] Bestor TH. The DNA methyltransferases of mammals. Hum Mol Genet 2000; 9(16): 2395-402.

[38] Huynh KD, Lee JT. X-chromosome inactivation: a hypothesis linking ontogeny and phylogeny. Nat Rev Genet 2005; 6(5): 410-8.

[39] Lewis A, Reik W. How imprinting centres work. Cytogenet Genome Res 2006; 113(1-4): 81-9.

[40] Reik W, Walter J. Genomic imprinting: parental influence on the genome. Nat Rev Genet 2001; 2(1): 21-32.

[41] Weaver IC, Cervoni N, Champagne FA, et al. Epigenetic programming by maternal behavior. Nat Neurosci 2004; 7(8): 84754.

[42] Milagro FI, Campion J, Garcia-Diaz DF, Goyenechea E, Paternain L, Martinez JA. High fat diet-induced obesity modifies the methylation pattern of leptin promoter in rats. J Physiol Biochem 2009; 65(1): 1-9.

[43] Campion J, Milagro FI, Fernandez D, Martinez JA. Diferential gene expression and adiposity reduction induced by ascorbic acid supplementation in a cafeteria model of obesity. J Physiol Biochem 2006; 62(2): 71-80. 
[44] Chang GQ, Gaysinskaya V, Karatayev O, Leibowitz SF. Maternal high-fat diet and fetal programming: increased proliferation of hypothalamic peptide-producing neurons that increase risk for overeating and obesity. J Neurosci 2008; 28(46): 12107-19.

[45] Plagemann A, Harder T, Brunn M, et al. Hypothalamic POMC promoter methylation becomes altered by early overfeeding: An epigenetic model of obesity and the metabolic syndrome. J Physiol 2009; 587(Pt 20): 4963-76.

[46] Muhlhausler BS, Adam CL, Findlay PA, Duffield JA, McMillen IC. Increased maternal nutrition alters development of the appetiteregulating network in the brain. FASEB J 2006; 20(8): 1257-9.

[47] Aagaard-Tillery KM, Grove K, Bishop J, et al. Developmental origins of disease and determinants of chromatin structure: maternal diet modifies the primate fetal epigenome. J Mol Endocrinol 2008; 41(2): 91-102.

[48] McCurdy CE, Bishop JM, Williams SM, et al. Maternal high-fat diet triggers lipotoxicity in the fetal livers of nonhuman primates. J Clin Invest 2009; 119(2): 323-35.

[49] Lillycrop KA, Phillips ES, Torrens C, Hanson MA, Jackson AA, Burdge GC. Feeding pregnant rats a protein-restricted diet persistently alters the methylation of specific cytosines in the hepatic PPAR alpha promoter of the offspring. Br J Nutr 2008; 100(2): 278-82.

[50] Vickers MH. Developmental programming and adult obesity: the role of leptin. Curr Opin Endocrinol Diabetes Obes 2007; 14(1): 17-22.

[51] Dolinoy DC, Weidman JR, Waterland RA, Jirtle RL. Maternal genistein alters coat color and protects Avy mouse offspring from obesity by modifying the fetal epigenome. Environ Health Perspect 2006; 114(4): 567-72.

[52] McMillen IC, Edwards LJ, Duffield J, Muhlhausler BS. Regulation of leptin synthesis and secretion before birth: implications for the early programming of adult obesity. Reproduction 2006; 131(3): 415-27.

[53] Campion J, Milagro FI, Martinez JA. Individuality and epigenetics in obesity. Obes Rev 2009; 10(4): 383-92.

[54] Dolinoy DC, Jirtle RL. Environmental epigenomics in human health and disease. Environ Mol Mutagen 2008; 49(1): 4-8.

[55] Gallou-Kabani C, Junien C. Nutritional epigenomics of metabolic syndrome: new perspective against the epidemic. Diabetes 2005; 54(7): 1899-906.

[56] Kaati G, Bygren LO, Edvinsson S. Cardiovascular and diabetes mortality determined by nutrition during parents' and grandparents' slow growth period. Eur J Hum Genet 2002; 10(11): 682-8.

[57] Feil R. Environmental and nutritional effects on the epigenetic regulation of genes. Mutat Res 2006; 600(1-2): 46-57.

[58] Lumey LH. Decreased birthweights in infants after maternal in utero exposure to the Dutch famine of 1944-1945. Paediatr Perinat Epidemiol 1992; 6(2): 240-53.

[59] Stein AD, Lumey LH. The relationship between maternal and offspring birth weights after maternal prenatal famine exposure: the Dutch Famine Birth Cohort Study. Hum Biol 2000; 72(4): 641-54.

[60] Martinez JA, Parra MD, Santos JL, Moreno-Aliaga MJ, Marti A, Martinez-Gonzalez MA. Genotype-dependent response to energyrestricted diets in obese subjects: towards personalized nutrition. Asia Pac J Clin Nutr 2008; 17 (Suppl 1): 119-22. 